\title{
The hardness profile as a tool to detect spurious stationary points in the potential energy surface
}

\author{
Miquel Torrent-Sucarrat, Josep M. Luis, ${ }^{\text {a) }}$ Miquel Duran, and Miquel Solàb) \\ Institut de Química Computacional and Departament de Química, Universitat de Girona, E-17071 Girona, \\ Catalonia, Spain
}

(Received 11 March 2004; accepted 24 March 2004)

\begin{abstract}
In the present work, we have computed the energy and hardness profiles for a series of inter and intramolecular conformational changes at several levels of calculation. All processes studied have in common the fact that the choice of a weak methodology or a poor basis set results in the presence of spurious stationary points in the energy profile. At variance with the energy profiles, the hardness profiles calculated as the difference between the vertical ionization potential and electron affinity always show the correct number of stationary points independently of the basis set and methodology used. For this reason, we have concluded that hardness profiles can be used to check the reliability of the energy profiles for those chemical systems that, because of their size, cannot be treated with high level ab initio methods. (c) 2004 American Institute of Physics. [DOI: 10.1063/1.1742793]
\end{abstract}

\section{INTRODUCTION}

The theoretical treatment of a chemical reaction requires detailed knowledge of the potential energy surface (PES) in terms of all internal degrees of freedom. There are, however, too many degrees of freedom to map out the full PES. To circumvent this problem, one attempts to locate the stationary (or critical) points on the surface, those places in the PES that have zero gradient. ${ }^{1}$ These stationary points can be of several types, depending on the nature of their second derivatives, the most chemically significant being the minima and the first-order saddle points. Minima on the PES can be identified with the classical picture of equilibrium structures of molecules, while first-order saddle points can be related to transition states. From the transition state down to the reactants and down to the products a reaction path is defined as the steepest descent path. If mass-weighted Cartesian coordinates are used, the path is called the intrinsic reaction path (IRP) ${ }^{2,3}$ The determination of minima, transition states, and the reaction paths connecting them is an important task of theoretical chemistry that allows to find out the thermochemistry and kinetics of the reactions and to discuss reaction mechanisms. ${ }^{4}$ The development of methods to locate the relevant stationary points on the PESs has brought in the last thirty years a complete revolution in the study of chemical reactions. ${ }^{5}$

However, equally important to the existence of methodology that makes possible the location of stationary points on the PES is the quest for principles, laws, theorems, postulates, or rules that rationalize the large body of available scattered information on chemical reactivity. Among the most fundamental chemical reactivity principles is the maximum hardness principle (MHP) ${ }^{6-8}$ which asserts that molecular systems at equilibrium tend to the state of highest hardness. The hardness ${ }^{7}$ is a measure of the resistance of a

\footnotetext{
a)Electronic mail: josepm.luis@udg.es

${ }^{b)}$ Electronic mail: miquel.sola@udg.es
}

chemical species to change its electronic configuration. Within the framework of the conceptual density functional theory (DFT) (Refs. 9 and 10) the hardness is given by

$$
\eta=\left(\frac{\partial^{2} E}{\partial N^{2}}\right)_{\nu(\mathbf{r})} .
$$

Here $N$ is the number of electrons, and $\nu(\mathbf{r})$ the potential of the nuclei and any external potential. On the basis of the MHP and the inverse relationship between the hardness and polarizability, ${ }^{11}$ Chattaraj and Sengupta ${ }^{12}$ formulate the minimum polarizability principle (MPP). The MPP affirms that the natural evolution of any system is towards a state of minimum polarizability.

According to the MHP (and the MPP), the variation of the hardness (and polarizability) of a chemical species along the reaction path, the so-called hardness (and polarizability) profiles, should go through a minimum (maximum) at the transition state and through maxima (minima) for reactants and products. A formal proof of the MHP was given by Parr and Chattaraj ${ }^{13}$ under the constraints that the electronic chemical potential and the external potential must remain constant upon distortion of the molecular structure. These are two severe constraints that are usually not fulfilled. However, relaxation of these constraints seems to be permissible and, in particular, it has been found that, for a number of chemical reactions, the MHP and MPP holds. ${ }^{14-24}$ Although the results that support the validity of MHP in chemical processes have been accumulating, examples in which the MHP and MPP fail have been also reported. ${ }^{25-32}$ The studies on hardness profiles carried out so far ${ }^{14-32}$ indicate that a stationary point (maximum or minimum) of the hardness profile close to the transition state of the PES is usually found, although it does not coincide exactly with the location of the transition state in the reaction path. The same situation is also generally found for minima. Thus, commonly the number of critical points in the hardness profile matches the number of stationary points in the energy profile along the reaction path. 
Although in recent years there have been enormous improvements in the computational techniques available for determining the stationary points on the PES, ${ }^{4}$ it still requires a great deal of effort to obtain the accuracy that is necessary to get PESs which fully agree with experimental observations. It has been found for a number of systems that the location of minima and transition states in the PES is exposed to dramatic changes depending on the methodology and basis set used. ${ }^{33-48}$ This is particularly true in the case of reactions involving weak inter and/or intramolecular interactions. In these reactions, the number and/or the nature of stationary points (minima or transition states) may change with the basis set and/or the method of calculation [for instance, by including or not the basis set superposition error (BSSE) in the calculation of the PES (Refs. 39, 40, 48)]. When this occurs, it is difficult to judge the reliability of the PES obtained without performing high level $a b$ initio calculations that, on the other hand, are unaffordable for large size systems. In this sense, there is a need for simple and computationally cheap methods that bring to light the presence of spurious stationary points on the calculated PES.

The aim of this work is to compute the energy and hardness profiles for several conformational changes to show that for a given reaction the hardness profile along the reaction path is less dependent on the method and basis set used than the energy profile. To this end, we have chosen a series of well-known chemical processes for which the use of a too much rigid basis set or a too simple method of calculation leads to wrong PESs. ${ }^{33-39}$ The results obtained will reveal that the hardness profile can be used as a good indicator of the presence of spurious stationary points on the calculated PES.

\section{COMPUTATIONAL DETAILS}

The evaluation of the exact hardness [Eq. (1)] is subject to the problem that the molecular or atomic energy is a discrete function of the number of electrons. ${ }^{49,50}$ Although exhaustive investigations have been conducted on the improvement of alternative methods to obtain accurate hardnesses, ${ }^{18,51-54}$ the most used operational equation of the hardness is still based on the finite difference approximation

$$
\eta=I-A,
$$

where $I$ and $A$ are the first vertical ionization potential and electron affinity of the neutral molecule, respectively. Equation (2) can be approximated in molecular orbital calculations by using the Koopmans' theorem $\left(I \approx-\varepsilon_{H}\right.$ and $A$ $\left.\approx-\varepsilon_{L}\right) .{ }^{55}$ For closed shell species one obtains

$$
\eta=\varepsilon_{L}-\varepsilon_{H},
$$

where $\varepsilon_{H}$ and $\varepsilon_{L}$ are the energies of the highest occupied molecular orbital (HOMO) and the lowest unoccupied molecular orbital (LUMO), respectively. Throughout the paper we refer to the hardness calculated from Eq. (2) as $\eta_{2}$ and the hardness obtained using the Koopmans' theorem as $\eta_{1}$.

We have calculated the energy and hardness $(E$ and $\eta)$ profiles for conformational changes of three molecules $\left(\mathrm{H}_{2} \mathrm{O}_{2}, \mathrm{~B}_{2} \mathrm{~F}_{4}\right.$, and $\left.\mathrm{Si}_{2} \mathrm{C}\right)$ and two molecular complexes $\left(\mathrm{H}_{2} \mathrm{O} \cdots \mathrm{HCl}\right.$ and $\left.\mathrm{HCCH} \cdots \mathrm{O}_{3}\right)$. The PESs of these molecules strongly depend on the level of calculation employed appearing spurious stationary points when the methodology and/or basis set used are not flexible enough. Figure 1 depicts the correct stationary points of the chemical systems investigated and the internal coordinates chosen to build the linear transit paths. For each reaction, these paths have been obtained by freezing at different values the selected internal coordinates given in Fig. 1 and optimizing the rest of internal coordinates. At each point of the linear transit path, we have calculated the $E$ and $\eta$ values needed to depict the corresponding profiles.

The geometry optimizations and hardness calculations have been performed at the Hartree-Fock (HF) ${ }^{56}$ hybrid density functional B3LYP, ${ }^{57}$ and second-order MøllerPlesset (MP2) (Ref. 58) methods using a series of Pople ${ }^{59}$ and Dunning's correlation-consistent basis sets. ${ }^{60}$ All these calculations have been carried out with the GAUSSIAN 98 package. ${ }^{61}$ The energy of the cationic and anionic doublet species, needed to obtain $I$ and $A$, has been computed within the unrestricted methodology at the geometry of the neutral systems, while the neutral singlet molecules have been calculated using the restricted formalism.

\section{RESULTS AND DISCUSSION}

This section is organized as follows: first, we present three examples of intramolecular conformational changes $\left(\mathrm{H}_{2} \mathrm{O}_{2}, \mathrm{~B}_{2} \mathrm{~F}_{4}\right.$, and $\left.\mathrm{Si}_{2} \mathrm{C}\right)$ and second, we discuss the energy and hardness profiles for conformational modifications in two intermolecular complexes $\left(\mathrm{H}_{2} \mathrm{O} \cdots \mathrm{HCl}\right.$ and $\mathrm{HCCH} \cdots \mathrm{O}_{3}$ ).

\section{A. Intramolecular conformational changes}

\section{1. $\mathrm{H}_{2} \mathrm{O}_{2}$}

In the literature there are excellent treatments of the torsional rotation of hydrogen peroxide. ${ }^{33,41}$ The role of the basis sets and correlation effects on the energy barriers for this conformational process was already investigated in detail by Cremer in 1978. ${ }^{33}$ Whereas the polarization functions are essential to obtain the correct torsional potential energy profile, the inclusion of electronic correlation plays no major role. Nevertheless, the electronic correlation is necessary to obtain accurate equilibrium geometries.

The $E, \eta_{1}$, and $\eta_{2}$ profiles for the linear transit path corresponding to the torsional rotation of hydrogen peroxide defined by the $\angle \mathrm{HOOH}$ angle computed at the B3LYP level using the 6-31G, 6-311++ G, 6-31+ $\mathrm{G}(d)$, and 6-311+ $+\mathrm{G}(3 d f, 3 p d)$ basis sets are depicted in Fig. 2. The potential energy profiles given by the $6-31+\mathrm{G}(d)$ and 6-311+ $+\mathrm{G}(3 d f, 3 p d)$ basis sets have the correct shape, presenting a minimum for a $\angle \mathrm{HOOH}$ angle of about $110^{\circ}$ [the experimental value $e^{62}$ is $\left.120 .(2)^{\circ}\right]$ and two transition states at $0^{\circ}$ and $180^{\circ}$, while the $6-31 \mathrm{G}$ and $6-311++\mathrm{G}$ basis sets yield wrong potential energy profiles. The B3LYP/6-311++G minimum is placed at about $150^{\circ}$ and presents a negligible barrier on the path to the $180^{\circ}$ transition state. The 6-31G PES has two stationary points instead of three, since the minimum is situated at $180^{\circ}$ and only the $0^{\circ}$ transition state 
<smiles>OO</smiles>
TS $C_{2 h}$<smiles>FB(F)B(F)F</smiles>

Minimum $D_{2 h}$<smiles>[SiH3]C1CC([SiH3])C1</smiles>

Minimum $C_{2 v}$<smiles>[X][C](O)Cl</smiles>

Minimum $C_{s}$

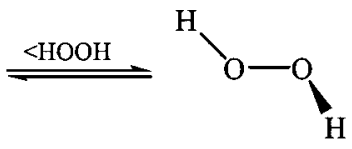

Minimum $C_{2}$

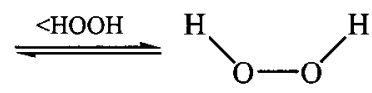

$\operatorname{TS} C_{2 v}$
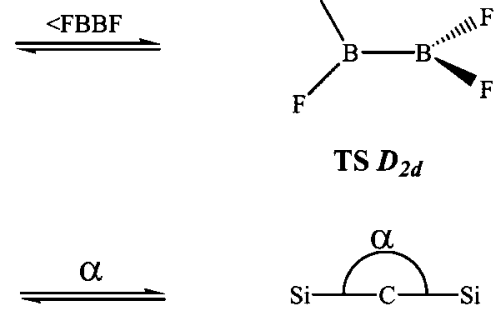

TS $C_{\infty} v$

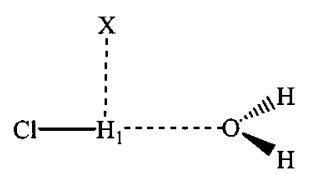

$\operatorname{TS} C_{2 v}$

FIG. 1. A schematic representation of the reactions studied with their correct stationary points and the internal coordinates chosen to computed the linear transit paths.

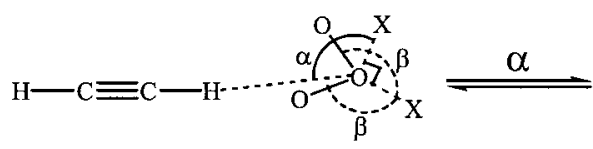

Minimum $C_{s}$

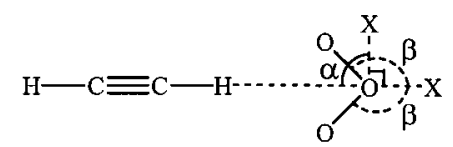

TS $C_{2 v}$ remains. Thus, the $6-31 \mathrm{G}$ basis set leads to a spurious planar trans structure for the equilibrium geometry of the $\mathrm{HOOH}$ species.

In contrast to the strong dependence of the energy profile on the basis set, the shape of the $\eta_{1}$ and $\eta_{2}$ profiles showed in Fig. 2 is nearly independent on the basis set. Both hardness profiles present always two minima at $0^{\circ}$ and $180^{\circ}$, while the maximum is placed at about $90^{\circ}$. Although for $\mathrm{HOOH}$ the absolute values of $\eta_{1}$ are about half of the $\eta_{2}$ values, their variations between the different stationary points are quite similar.

The rotation of hydrogen peroxide follows the MHP. ${ }^{63}$ The minima and maximum of hardness indicate the presence of two transition states and an equilibrium structure, respectively. Then, the number of the stationary points in the energy and hardness profiles is the same. This is true for all basis sets but for the 6-31G one. In this case, the hardness profile has the right number and type of stationary points, while the energy profile is wrong. Thus, divergence between the number of stationary points of the energy and hardness profiles can be use to detect spurious stationary points in the PES. It is well known that the location of the hardness stationary points does not coincide with the location of the energy stationary points. Nevertheless, it is worth noting that the $6-311++\mathrm{G}(3 d f, 3 p d)$ profile, which is the profile obtained with the largest basis set among those presented in Fig. 2, lead to the smallest difference between the position of the hardness and energy stationary points.

As it was pointed by Chandra and Uchimaru, ${ }^{30}$ and pre- viously observed by Makov, ${ }^{64}$ all symmetric-breaking displacements about a symmetric configuration of the nuclei will necessarily increase or decrease the energy and hardness and therefore the symmetric configurations must be stationary points of both potential energy and hardness (throughout the paper, we refer to the points of a profile that accomplish this condition as symmetric stationary points). For this reason, the existence of the $0^{\circ}$ and $180^{\circ}$ symmetric stationary points of the energy and hardness profile $\mathrm{HOOH}$ (i.e., the cis and trans planar structures) for all methods and basis sets can be predicted without calculations. This is not the case for the $110^{\circ}$ stationary point. Indeed, this minimum is not found in the B3LYP/6-31G energy profile. On the contrary, and because hardness profiles are more stable to the change of method and basis set than energy profiles, this stationary point is detected in the hardness profile. Therefore, the hardness profile can be useful to denote the existence of nonsymmetric stationary points, which can not be predicted from symmetry arguments. Furthermore, the hardness profile also indicates that the $0^{\circ}$ and $180^{\circ}$ symmetric stationary points must belong to the same type (i.e., minima or transition states). Thus, for the $6-31 \mathrm{G}$ case, the hardness profile points out that the nature of either the $0^{\circ}$ or the $180^{\circ}$ stationary points in the energy profile is incorrect.

The $E, \eta_{1}$, and $\eta_{2}$ profiles for the torsional rotation of $\mathrm{HOOH}$ computed at the B3LYP level with the STO-3G, $3-21 \mathrm{G}, 6-311 \mathrm{G}(d, p), 6-311++\mathrm{G}(d, p)$, and aug-cc-pVTZ basis sets, and at the MP2 level with the 6-31G and 6-311 $++\mathrm{G}(d, p)$ basis sets are provided as supporting informa- 


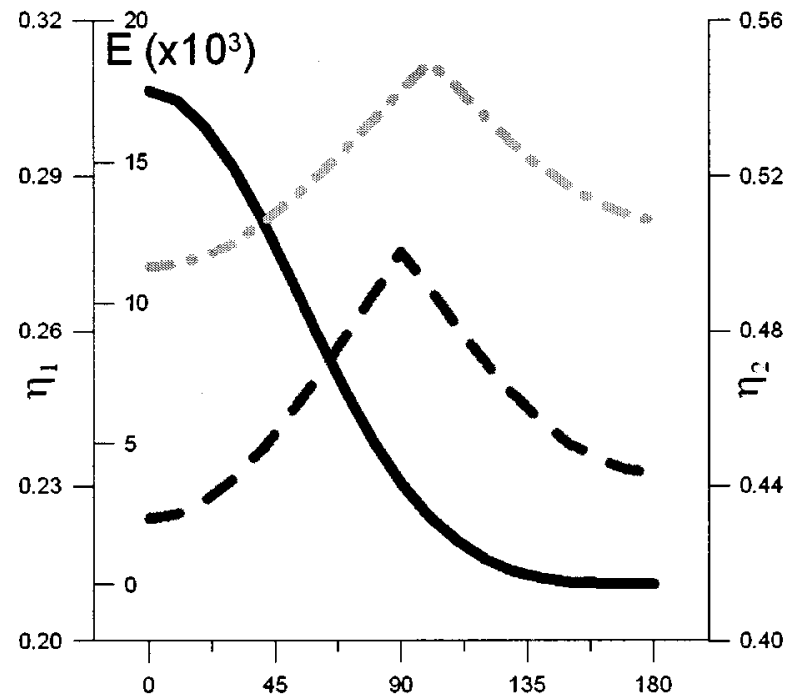

a) B3LYP/6-31G

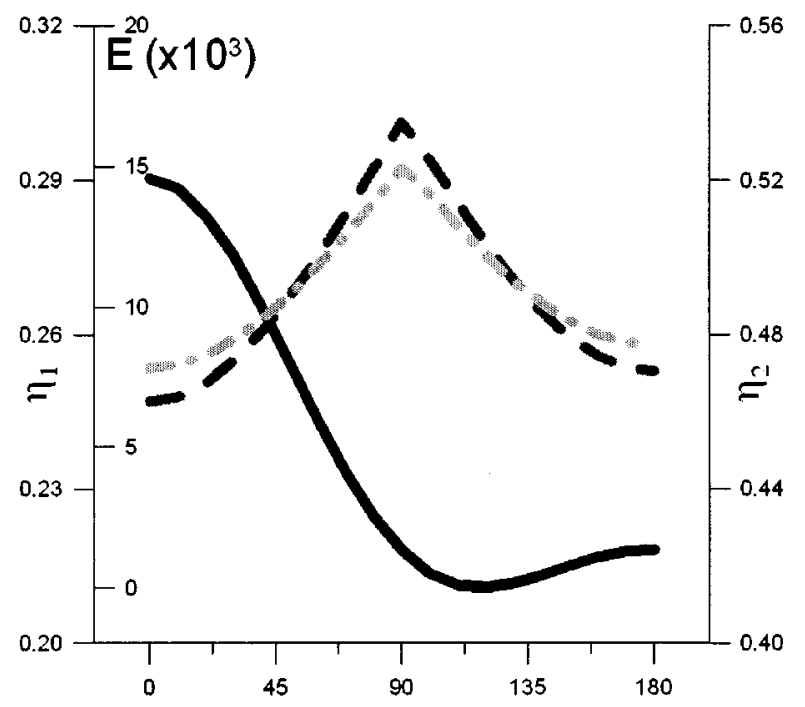

c) $B 3 L Y P / 6-31+G(d)$

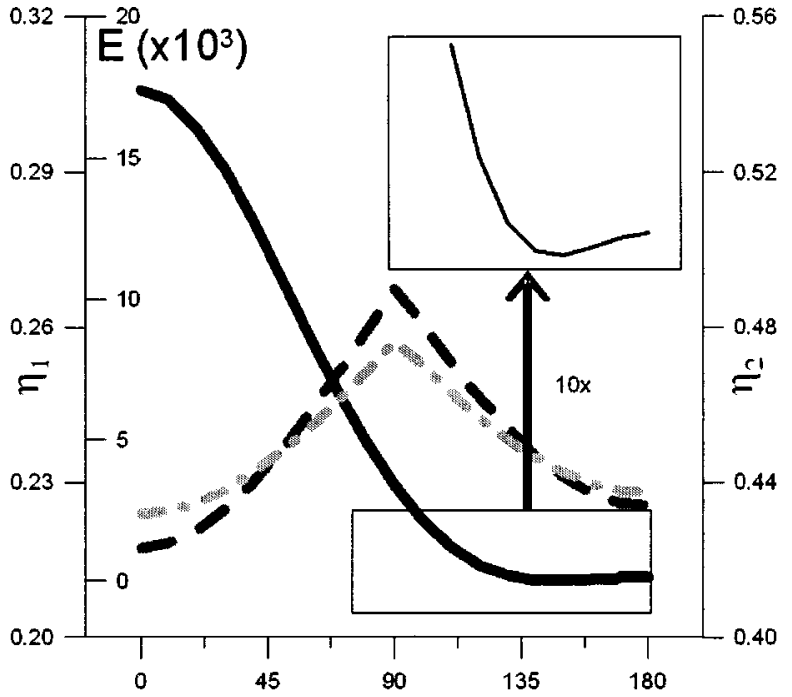

b) B3LYP/6-311++G

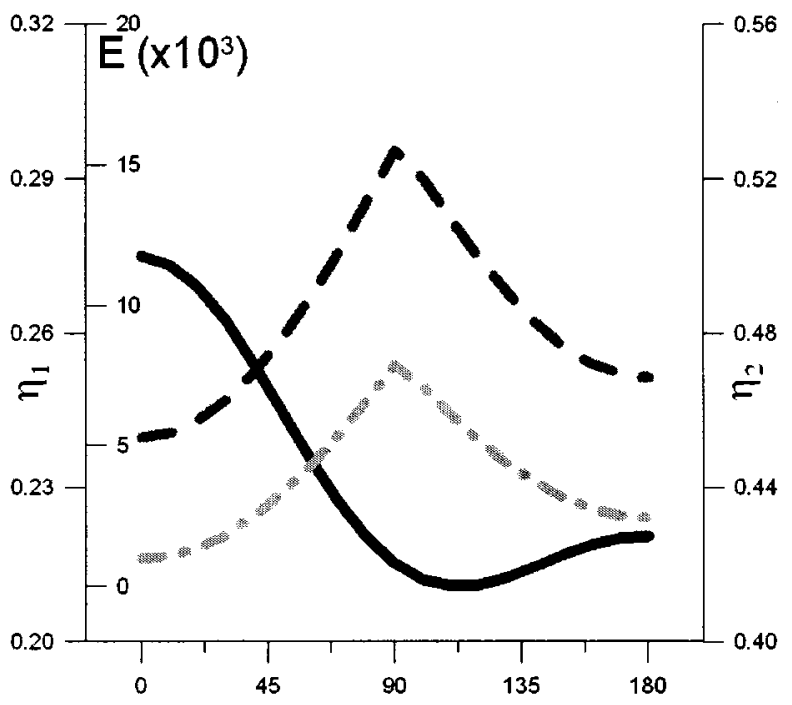

d) B3LYP/6-311++G(3df,3pd)

FIG. 2. Profiles of the relative energies (solid line) and hardness ( $\eta_{1}$ dashed line and $\eta_{2}$ dot-dashed line) calculated for the internal rotation of $\mathrm{H}_{2} \mathrm{O}_{2}$. The hardness values and the relative energies are given in a.u.

tion. The hardness profile has the correct shape for all methods and basis sets, albeit the energy profile presents spurious stationary points for the STO-3G and 3-21G basis sets with the B3LYP method and for the 6-31G basis set at the MP2 level. $^{65}$

\section{2. $B_{2} F_{4}$}

Recent calculations performed by Li and Fan highlighted the great difficulty that different theoretical methods experience to determine the equilibrium structure of the $\mathrm{B}_{2} \mathrm{~F}_{4}$ molecule in its ground state. ${ }^{34}$ The comparison of experimental vibrational spectroscopy and theoretical data shows that the ground state equilibrium geometry of the $\mathrm{B}_{2} \mathrm{~F}_{4}$ molecule has the $D_{2 h}$ eclipsed structure, while the staggered geometry with $D_{2 d}$ symmetry is a transition state. However, the energy profile around the rotation of the $\mathrm{B}-\mathrm{B}$ bond is so flat that the
B3LYP method does not provide the right profile even when large basis sets such as the cc-pVQZ are used.

In Fig. 3, we present the $E, \eta_{1}$, and $\eta_{2}$ profiles for the linear transit path corresponding to the rotation around the $\mathrm{B}-\mathrm{B}$ bond of the $\mathrm{B}_{2} \mathrm{~F}_{4}$ species calculated at the B3LYP level with the 6-31G, 6-311G $(d)$, cc-pVDZ, and cc-pVTZ basis sets. The $0^{\circ}$ and $180^{\circ}$ symmetric points correspond to the eclipsed structure, whereas the staggered geometry is located at $90^{\circ}$. The energy profiles obtained with the 6-31G and cc-pVDZ basis sets present these two stationary points, the $D_{2 h}$ geometry being a minimum and the $D_{2 d}$ structure a transition state. These two basis sets lead to the correct energy profile because the incorrect description of the chemical system given by the B3LYP method is fortuitously compensated by the relative inflexibility of the basis set used. Indeed, the 6-311G $(d)$ and cc-pVTZ basis sets give wrong 


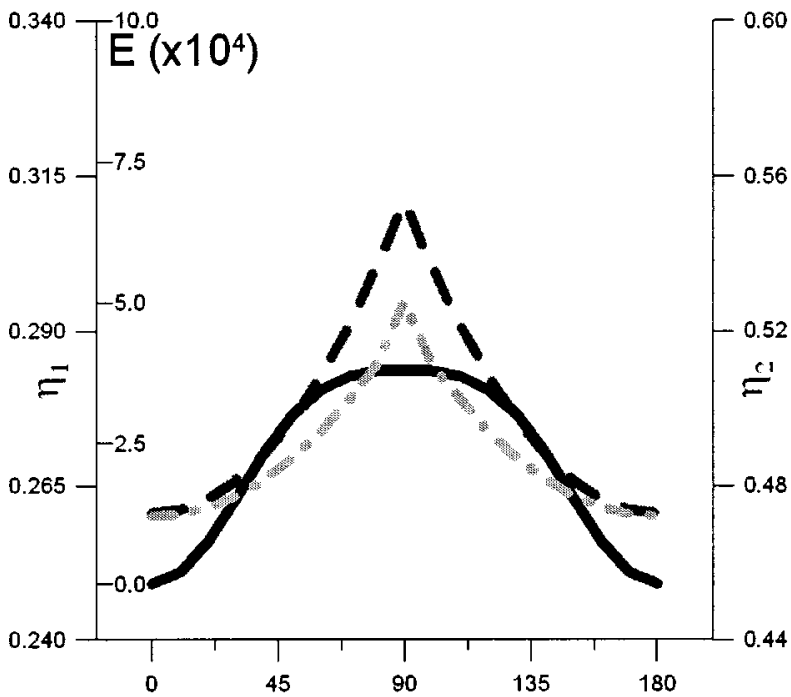

a) B3LYP/6-31G

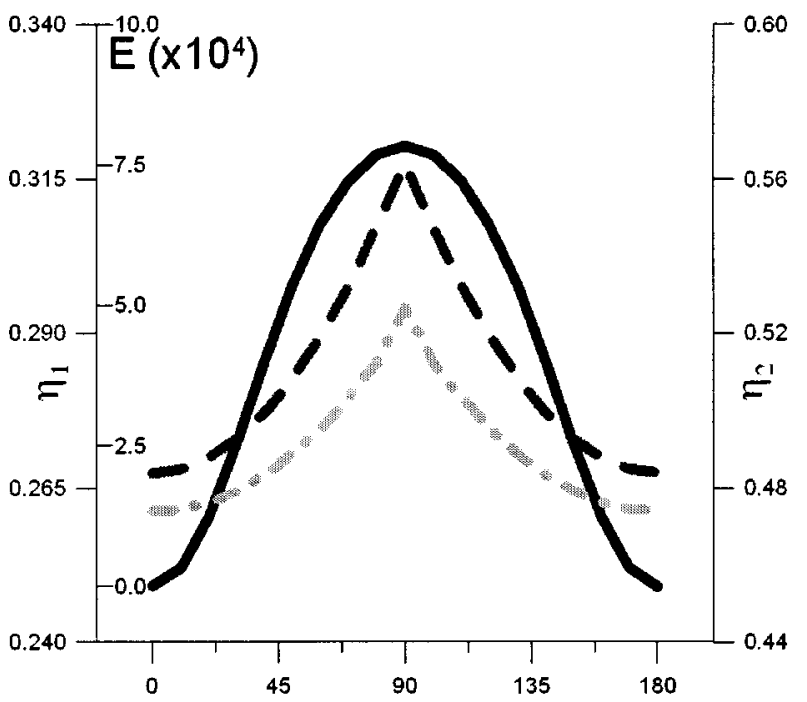

c) B3LYP/cc-pVDZ

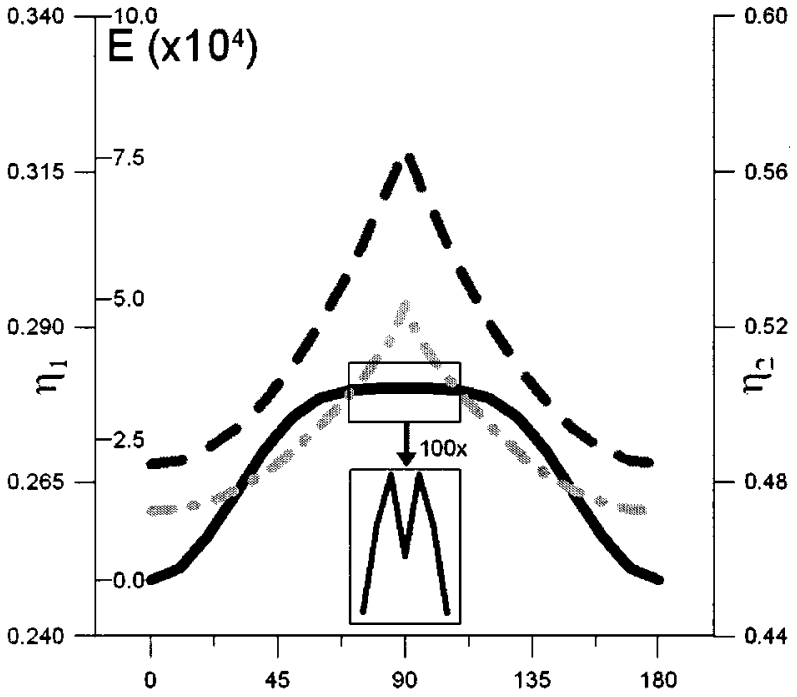

b) B3LYP/6-311G(d)

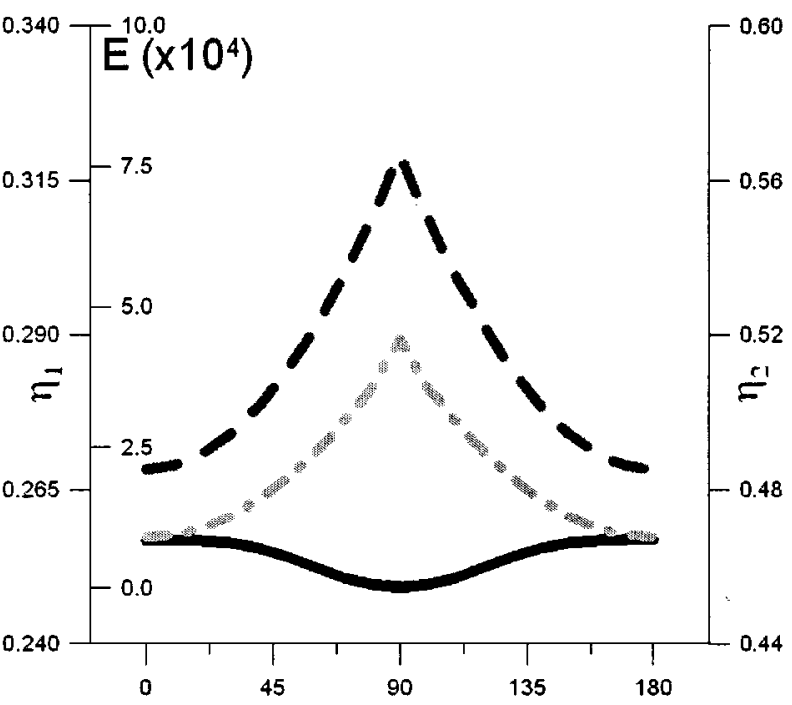

d) B3LYP/cc-pVTZ

FIG. 3. Profiles of the relative energies (solid line) and hardness ( $\eta_{1}$ dashed line and $\eta_{2}$ dot-dashed line) calculated for the rotation of $\mathrm{B}_{2} \mathrm{~F}_{4}$ around the $\mathrm{B}-\mathrm{B}$ bond. The hardness values and the relative energies are given in a.u.

energy profiles despite their higher quality. For the cc-pVTZ basis set, the eclipsed geometry is a transition state and the staggered geometry is a minimum. Finally, for the 6-311G $(d)$ basis set, the two symmetric stationary points corresponding to the eclipsed and staggered structures are minima and there is a nonsymmetric transition state between them.

Remarkably, the shape of the hardness profile is invariant for the four basis sets studied, indicating only the presence of the two symmetric stationary points. As can be seen in Fig. 3, the rotation around the $\mathrm{B}-\mathrm{B}$ bond of the $\mathrm{B}_{2} \mathrm{~F}_{4}$ molecule does not follow the MHP. This is not particularly surprising as we have mentioned in the Introduction. ${ }^{25-32}$ However, the number of stationary points in the hardness profile coincides with the number of stationary points in the correct energy profile. Thus, the comparison of the hardness and energy profiles points out that the nonsymmetric transition state found with the $6-311 \mathrm{G}(d)$ basis set is spurious. Unfortunately, the hardness profile is unable to indicate the incorrect nature of the stationary points in the cc-pVTZ energy profile, since both the energy and hardness profiles have the same number of stationary points. This would be only possible if we could know a priori whether the reaction follows or not the MHP. However, it is worth to notice that whereas there is a drastic difference between the energy profiles obtained with the cc-pVDZ and cc-pVTZ basis set, the hardness profiles calculated with both basis sets are nearly identical.

The $E, \eta_{1}$, and $\eta_{2}$ profiles for the rotation around the $\mathrm{B}-\mathrm{B}$ bond of the $\mathrm{B}_{2} \mathrm{~F}_{4}$ species calculated at the HF, B3LYP, and MP2 levels with the 6-31G $(d)$ basis set are drawn in Fig. 4. The HF energy profile presents a minimum in the $D_{2 d}$ 


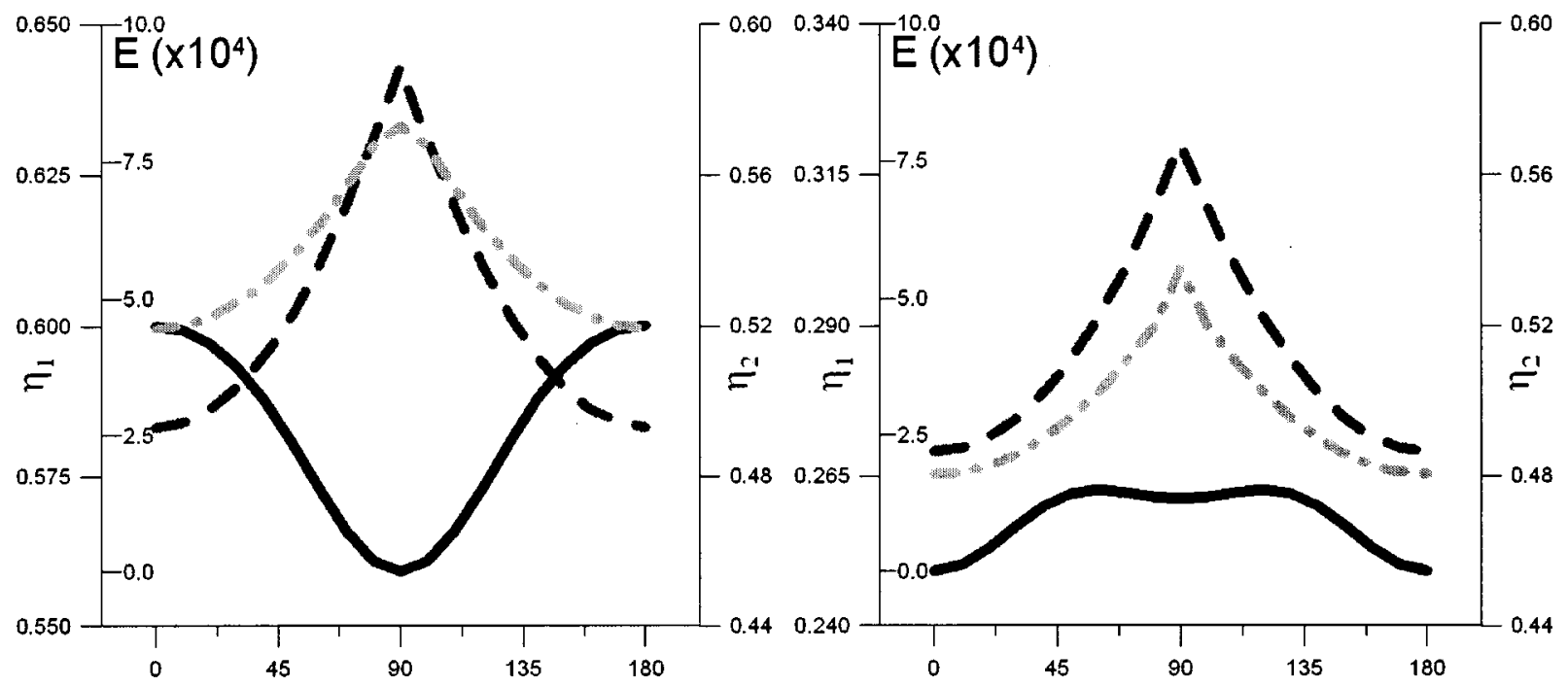

a) HF/6-31G(d)

b) B3LYP/6-31G(d)

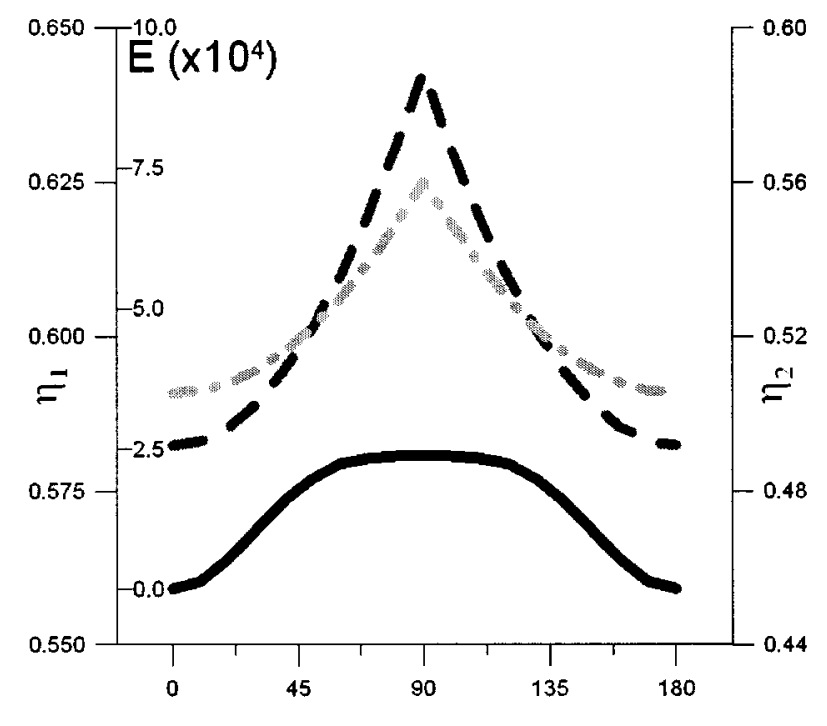

c) $M P 2 / 6-31 G(d)$

FIG. 4. Profiles of the relative energies (solid line) and hardness ( $\eta_{1}$ dashed line and $\eta_{2}$ dot-dashed line) calculated for the rotation of $\mathrm{B}_{2} \mathrm{~F}_{4}$ around the $\mathrm{B}-\mathrm{B}$ bond. The hardness values and the relative energies are given in a.u.

structure and a transition state in the $D_{2 h}$ geometry. On the contrary, the MP2 energy profile has just the opposite shape (i.e., the correct shape). At the B3LYP level, both structures are minima and then there is a nonsymmetric transition state between them. The hardness profiles obtained at the three levels of theory have the same shape. ${ }^{65}$ Thus, for $\mathrm{B}_{2} \mathrm{~F}_{4}$, the number and type of the stationary points of the hardness profile is always the same irrespective of the basis set and $a b$ initio method used. Again, although the hardness profile cannot detect the incorrect nature of the symmetric $\mathrm{HF} / 6-31 \mathrm{G}(d)$ stationary points, it is an excellent tool to point out that the nonsymmetric transition states found in the B3LYP/6-31G $(d)$ profile are spurious.

The $E, \eta_{1}$, and $\eta_{2}$ profiles for the rotation around the $\mathrm{B}-\mathrm{B}$ bond of $\mathrm{B}_{2} \mathrm{~F}_{4}$ computed at the HF and MP2 levels with the 6-31G, 6-311G $(d)$, cc-pVDZ, and cc-pVTZ basis sets can be found in the supporting information. The energy profiles calculated at the HF/6-31G, HF/6-311(d), and MP2/6$31 \mathrm{G}$ levels are wrong. The hardness profiles always present the same shape for all methods and basis sets checked.

\section{3. $\mathrm{Si}_{2} \mathrm{C}$}

Katafi et al. ${ }^{66}$ determined experimentally that the $\mathrm{Si}_{2} \mathrm{C}$ ground state structure has $C_{2 v}$ symmetry. This result was confirmed later theoretically by Grev and Schaefer. ${ }^{35}$ In their calculations at the CISD/DZ+2P level, the minimum for the ground state of the $\mathrm{Si}_{2} \mathrm{C}$ molecule occurs at $R_{\mathrm{Si}-\mathrm{C}}$ $=1.686 \AA$ and $\theta_{\mathrm{Si}-\mathrm{C}-\mathrm{Si}}=120.4^{\circ}$. Nevertheless, at the $\mathrm{HF} / \mathrm{DZ}+\mathrm{P}$ level the $C_{2 v}$ structure of $\mathrm{Si}_{2} \mathrm{C}$ collapses to the linear geometry. 


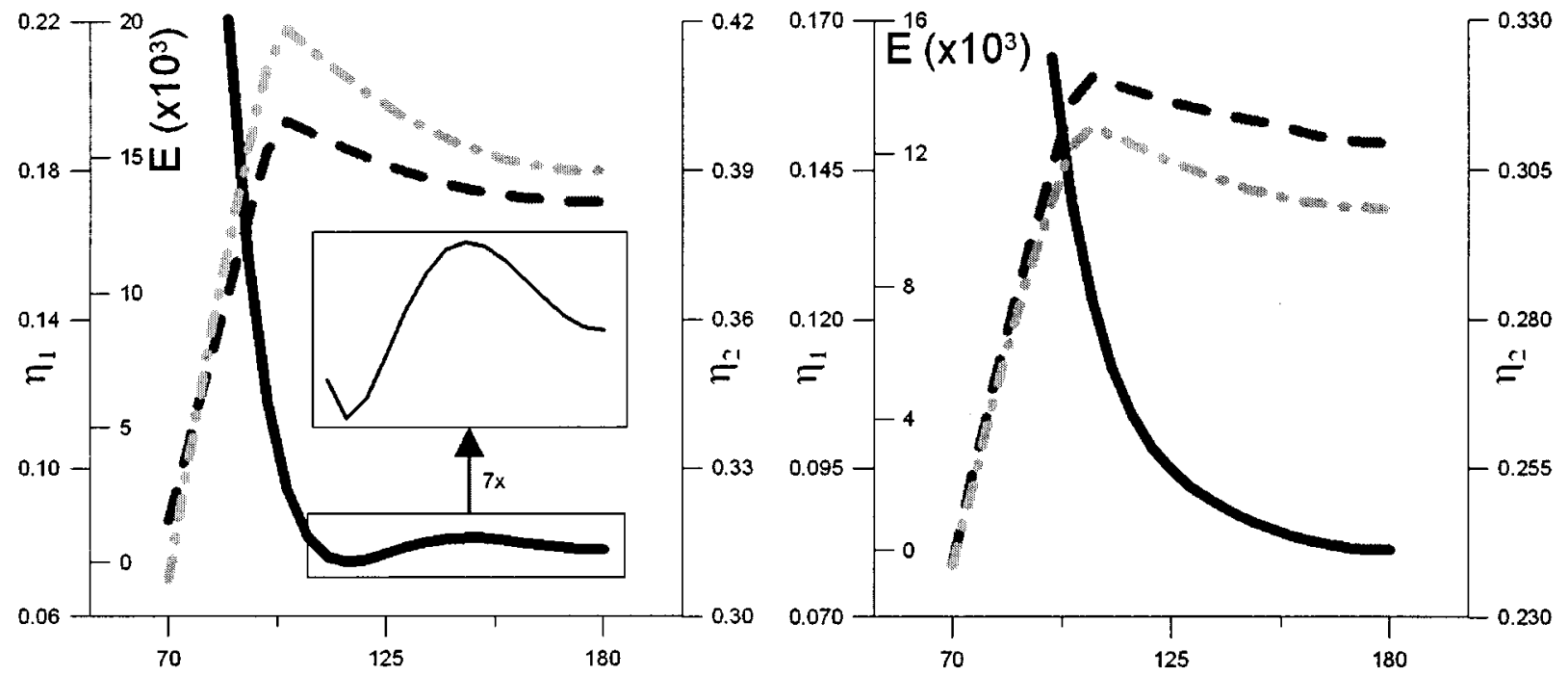

a) B3LYP/STO-3G

b) B3LYP/6-311+G(d)

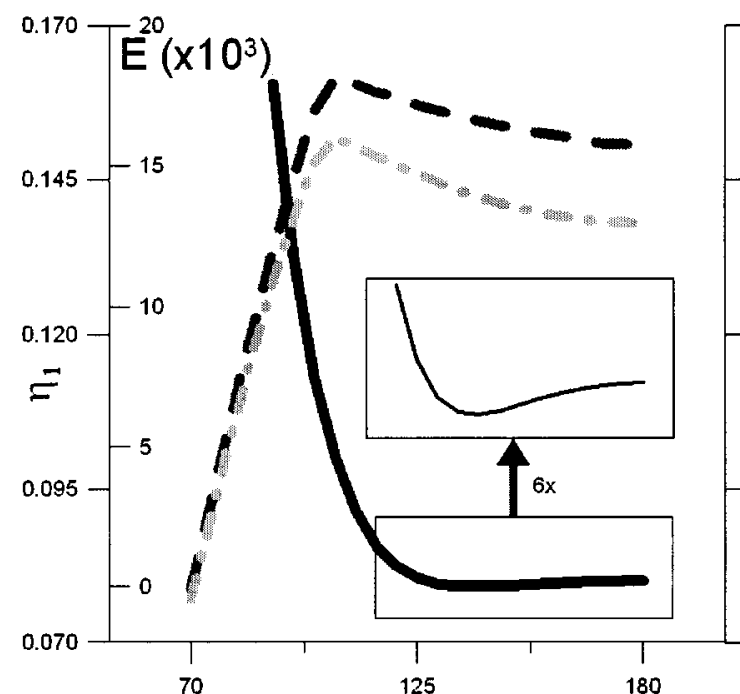

c) $B 3 L Y P / 6-311+G(3 d f)$

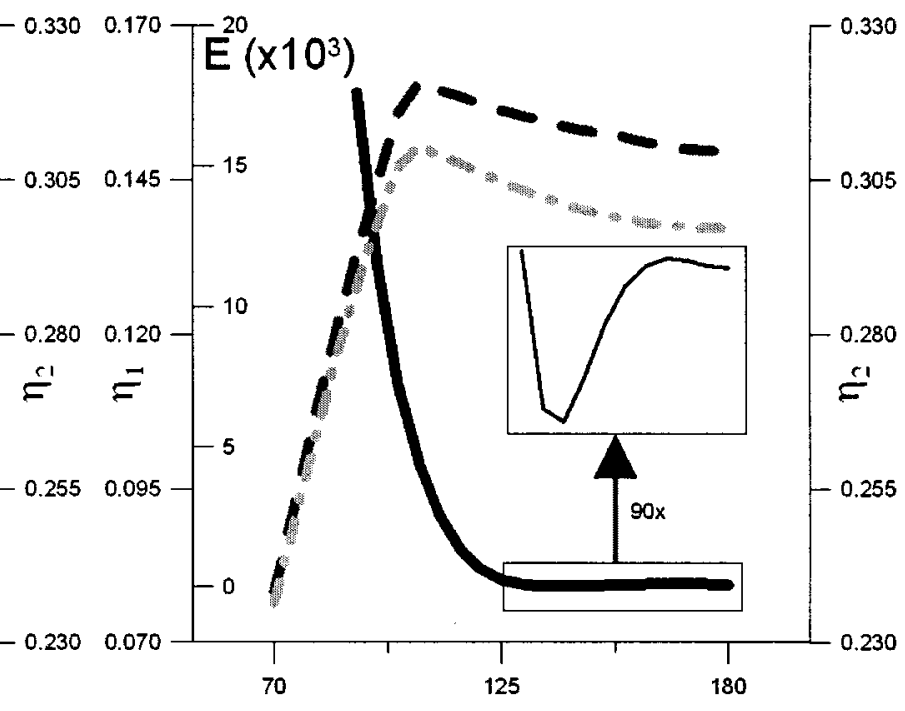

d) B3LYP/aug-cc-pVTZ

FIG. 5. Profiles of the relative energies (solid line) and hardness ( $\eta_{1}$ dashed line and $\eta_{2}$ dot-dashed line) calculated for the linear transit path described by the $\angle \mathrm{SiCSi}$ angle. The hardness values and the relative energies are given in a.u.

Figure 5 depicts the energy and hardness profiles along the linear transit path defined by the $\angle \mathrm{SiCSi}$ angle computed at the B3LYP level using the STO-3G, 6-311+ $\mathrm{G}(d)$, $6-311+\mathrm{G}(3 d f)$, and aug-cc-pVTZ basis sets. Only the $6-311+\mathrm{G}(3 d f)$ basis set leads to the correct energy profile with a symmetric transition state at $180^{\circ}$ and a nonsymmetric minimum at about $140^{\circ}$. The STO-3G and aug-cc-pVTZ energy profiles, although presenting a nonsymmetric minimum, have a symmetric spurious minimum instead of the symmetric transition state at $180^{\circ}$. Thus the energy profiles of these two basis sets have also a spurious nonsymmetric transition state between the nonsymmetric and symmetric minima. For the aug-cc-pVTZ basis set the energy difference between the linear minimum and the spurious transition state is very small $\left(4.1 \times 10^{-6}\right.$ a.u. $)$, but we have checked that the symmetric structure is a minimum by computing the har- monic vibrational frequencies. The $6-311+\mathrm{G}(d)$ energy profile shows a unique stationary point, which is a spurious symmetric minimum.

The intramolecular conformational change due to variation of the $\angle \mathrm{SiCSi}$ angle follows the MHP. Then, the symmetric transition state and the nonsymmetric energy minimum are indicated, respectively, by a minimum and a maximum on the hardness profile. Once more the hardness profiles have the same shape for the four basis sets analyzed. The comparison between the hardness and energy profiles obtained with the STO-3G and aug-cc-pVTZ basis sets clearly shows that their energy profiles are wrong since they have three stationary points instead of two as indicated by the hardness profile, which also reveals the spuriousness of the $6-311+\mathrm{G}(d)$ symmetric minimum. In this particular sys- 


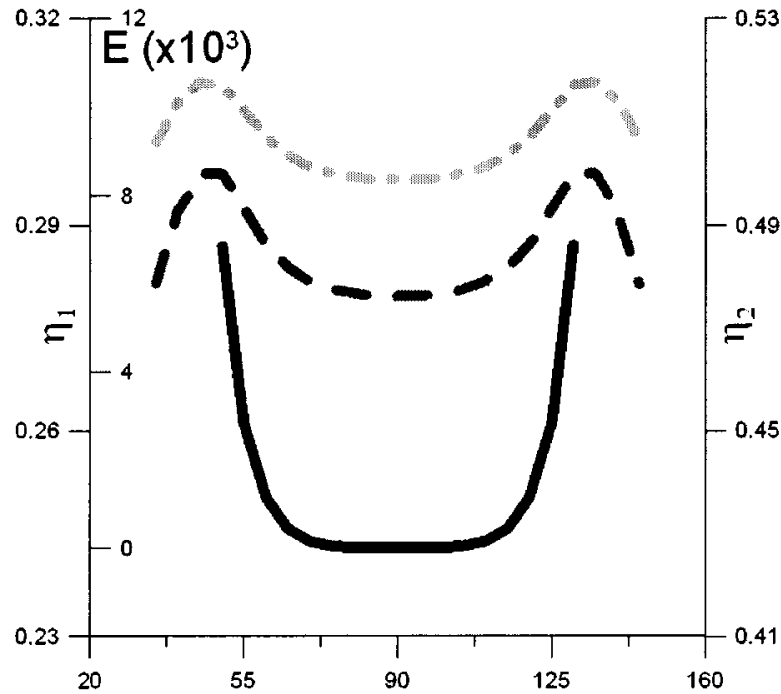

a) B3LYP/6-31G

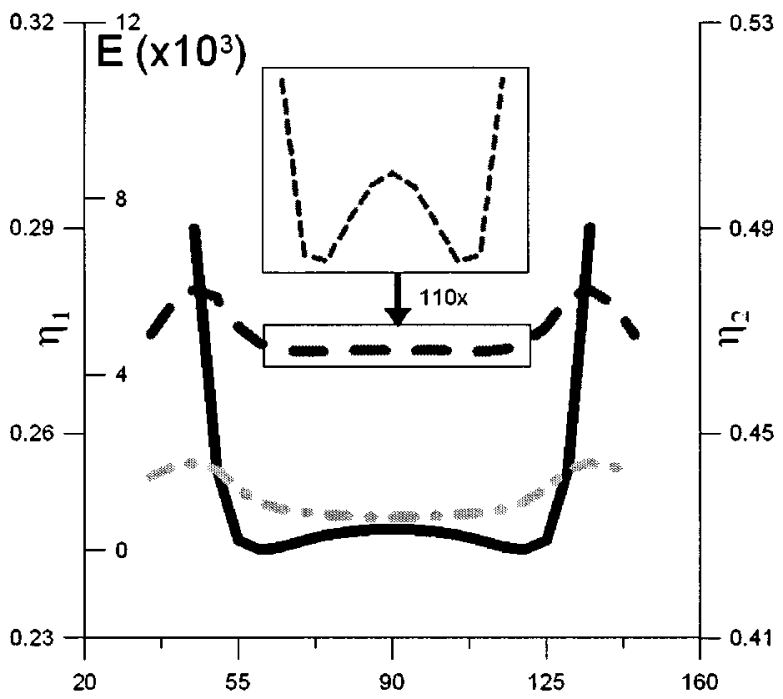

c) B3LYP/6-311++G(3df,3pd)

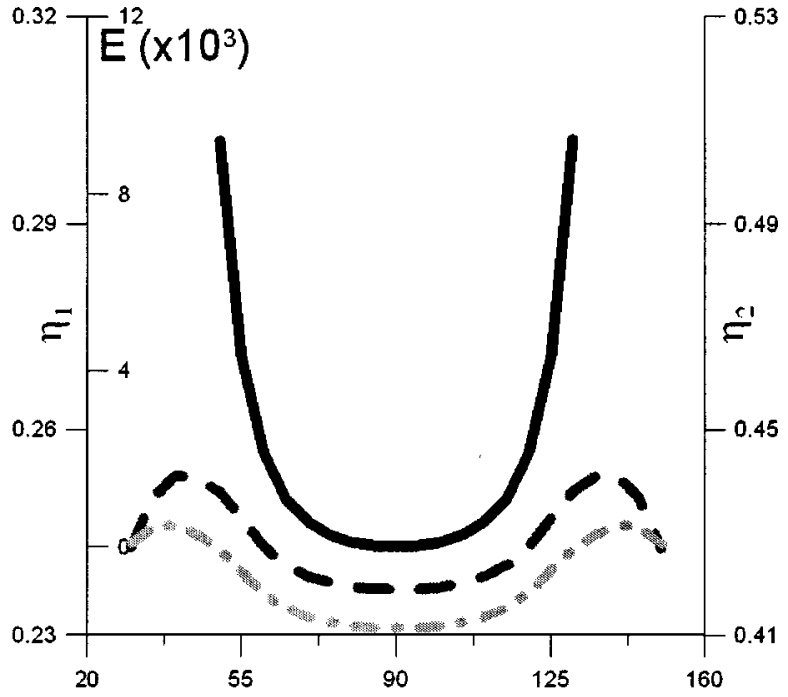

b) B3LYP/6-311++G

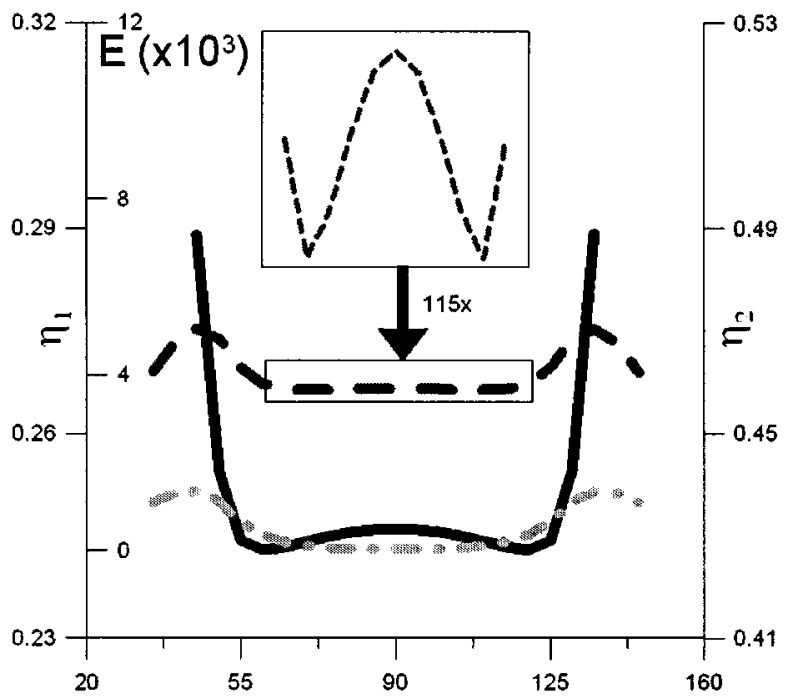

d) B3LYP/aug-cc-pVDZ

FIG. 6. Profiles of the relative energies (solid line) and hardness ( $\eta_{1}$ dashed line and $\eta_{2}$ dot-dashed line) calculated by changing the $\angle \mathrm{HOH} \mathrm{X}_{1}$ angle of the $\mathrm{H} 2 \mathrm{O} \cdots \mathrm{HCl}$ dimer. The hardness values and the relative energies are given in a.u.

tem, we know that necessarily the energy must increase when starting from the $C_{2 v}$ equilibrium geometry the $\angle \mathrm{SiCSi}$ angle decreases. Therefore the hardness profile completely determines the correct number and nature of the stationary points of the energy profile. It is worth noting that this is at variance with what we have found for the $\mathrm{B}_{2} \mathrm{~F}_{4}$ energy and hardness profiles at the B3LYP/cc-pVTZ level of theory. In that case, it was not possible to define the nature of the stationary points from the hardness profile alone. Finally, the $E, \eta_{1}$, and $\eta_{2}$ profiles calculated with the $3-21 \mathrm{G}, 6-31 \mathrm{G}$, $6-31+\mathrm{G}(d), 6-311+\mathrm{G}$, and $6-311 \mathrm{G}(d)$ basis sets at the B3LYP level are available in the supporting information. The hardness and energy profiles obtained with these five basis sets have the same shape than those obtained with the $6-311+\mathrm{G}(d)$ basis set.

\section{B. Intermolecular conformational changes}

\section{1. $\mathrm{H}_{2} \mathrm{O} \cdots \mathrm{HCl}$}

According to Kisiel et al., the experimental rotational constants of the $\mathrm{H}_{2} \mathrm{O} \cdots \mathrm{HCl}$ complex imply a nonplanar geometry for this hydrogen bonded dimer with $C_{s}$ symmetry. ${ }^{37}$ This experimental result was confirmed from BSSE corrected $a b$ initio results at the MP2/aug-cc-pVDZ level. ${ }^{37}$ However, if the theoretical calculations are performed with a basis set without polarization functions the equilibrium geometry obtained is planar with $C_{2 v}$ symmetry. ${ }^{36}$

Figure 6 represents the energy and hardness profiles computed at the B3LYP level using the 6-31G, 6-311+ $+\mathrm{G}, 6-311++\mathrm{G}(3 d f, 3 p d)$, and aug-cc-pVTZ basis sets for the linear transit path obtained by changing the angle 
between the water plane and a perpendicular axis to the $\mathrm{HCl}$ bond (the $\angle \mathrm{HOH}_{1} \mathrm{X}$ angle in Fig. 1). When the value of the $\angle \mathrm{HOH}_{1} \mathrm{X}$ angle is $90^{\circ}$ all the atoms of the complex are in the same plane and the system has $C_{2 v}$ symmetry, whereas for the other values of the $\angle \mathrm{HOH}_{1} \mathrm{X}$ angle the symmetry of the molecule is decreased to $C_{s}$. In concordance with previous literature results, ${ }^{37}$ the two basis sets with polarization functions present a symmetric transition state and two equivalent nonsymmetric minima at about $55^{\circ}$ and $125^{\circ}$. On the contrary, the $6-31 \mathrm{G}$ and $6-311++\mathrm{G}$ energy profiles show a unique spurious symmetric minimum at $90^{\circ}$.

The shape of the $\eta_{2}$ profiles in Fig. 6 for the four basis sets considered presents a minimum at the symmetric stationary point at $90^{\circ}$ and two maxima close to the nonsymmetric energy minima, thus following the MHP and reinforcing the idea that the hardness profiles are less dependent on the method and basis set than the energy profiles. Apparently, the shape of the $\eta_{1}$ profiles is identical to that of the $\eta_{2}$ profiles. However, when the hardness scale is augmented one hundred times, it is possible to observe that the 6-311+ $+\mathrm{G}(3 d f, 3 p d)$ and aug-cc-pVTZ $\eta_{1}$ profiles present a maximum instead of a minimum at the $C_{2 v}$ geometry and two extra equivalent nonsymmetric minima very close to the $C_{2 v}$ geometry (i.e., five stationary points). At this point we want to remind the reader that, as it was explained in the previous section, $\eta_{1}$ is just an approximation to $\eta_{2}$ and, therefore, the $\eta_{2}$ profiles are, in principle, of better-quality than the $\eta_{1}$ profiles. Thus, in case the shape of the two profiles does not coincide, the $\eta_{2}$ profile should be regarded as the correct one. Furthermore, if we use the IRP in place of the linear transit path defined by the $\angle \mathrm{HOH}_{1} \mathrm{X}$ angle to depict the $6-311++\mathrm{G}(3 d f, 3 p d)$ and aug-cc-pVTZ $\eta_{1}$ profiles, the two nonsymmetric minima disappear. Thus, using the IRP, the shape of the $\eta_{1}$ profile is analogous to that of the $\eta_{2}$ profile with a symmetric minimum and two nonsymmetric maxima for the four basis sets.

The supporting information contains the $E, \eta_{1}$, and $\eta_{2}$ profiles obtained at the B3LYP level with the STO-3G, $3-21 \mathrm{G}, 6-31+\mathrm{G}(d), 6-311 \mathrm{G}(d, p)$, and $6-311++\mathrm{G}(d, p)$ basis sets. The $E$ and $\eta_{2}$ profiles are always the correct (with three stationary points), but the $\eta_{1}$ profiles obtained with the STO-3G, 3-21G, and 6-311G $(d, p)$ basis sets have five stationary points instead of three. Nevertheless, like for the $6-311++\mathrm{G}(3 d f, 3 p d)$ and aug-cc-pVTZ $\eta_{1}$ profiles, the two nonsymmetric minima vanish when the IRP is used instead of the linear transit path defined by the $\angle \mathrm{HOH}_{1} \mathrm{X}$ angle.

\section{2. $\mathrm{HCCH} \cdots \mathrm{O}_{3}$}

The theoretical study of several complexes containing $\mathrm{C}-\mathrm{H} \cdots \mathrm{O}$ performed by Turi and Dannenberg ${ }^{38}$ showed that the optimized equilibrium geometry of the complexes involving ozone differs significantly depending on the basis set used. At the HF and MP2 levels, the equilibrium geometry of $\mathrm{HCCH} \cdots \mathrm{O}_{3}$ has $C_{2 v}$ symmetry with the $6-31 \mathrm{G}(d, p)$ basis set, whereas with the $\mathrm{D} 95++\mathrm{G}$ basis set it has a lower $C_{s}$ symmetry. Salvador et al. ${ }^{39}$ evidenced some years later that the spurious $C_{2 v}$ equilibrium geometry obtained with the $6-31 \mathrm{G}(d, p)$ basis set was due to the BSSE. Thus, these au- thors found that when the BSSE is corrected during the geometry optimization process, both the $6-31 \mathrm{G}(d, p)$ and $\mathrm{D} 95++\mathrm{G}$ basis sets predict the same $C_{s}$ equilibrium geometry. ${ }^{39}$

Figure 7 depicts the $E$ and $\eta_{1}$ profiles for the linear transit path defined by the angle between a line perpendicular to the $C_{2}$ axis of the ozone molecule and the line that connects the $\mathrm{H}$ with the central oxygen of the $\mathrm{O}_{3}$ molecule (the $\angle \mathrm{HOX}$ angle in Fig. 1) computed at the B3LYP level using the 6-31G, 6-31G $(d, p), 6-311++\mathrm{G}(d, p)$, and aug-ccpVDZ basis sets. The dimer has $C_{s}$ symmetry for all $\angle \mathrm{HOX}$ angles, except for the $\angle \mathrm{HOX}$ angle of $90^{\circ}$ where the complex has $C_{2 v}$ symmetry. In complete agreement with previous HF and MP2 results, ${ }^{38,39}$ the B3LYP/6-31G $(d, p)$ energy profile has a spurious minimum with $C_{2 v}$ geometry. A similar energy profile with a unique symmetric stationary point is obtained using the 6-31G basis set. On the contrary, the two largest basis sets yield energy profiles with two equivalent nonsymmetric minima at about $70^{\circ}$ and $110^{\circ}$ and a symmetric transition state connecting them at $90^{\circ}$.

The HOMO and HOMO-1 orbitals of the $\mathrm{HCCH} \cdots \mathrm{O}_{3}$ complex are quasidegenerate. For such a system, it is difficult to evaluate accurately with a single determinant based method like B3LYP or HF the energy of the system with $N-1$ electrons that is needed to calculate $\eta_{2}$. Actually, at the B3LYP level we have been unable to obtain a converged wave function, and at the HF level we obtain an $S^{2}$ expected value of about 1.75 , far from the correct value of 0.75 , which denotes the deficiency of the computed wave function. This problem could be overcome using a multiconfiguration method like MCSCF, but this is out of the scope of this paper. For this reason, we have calculated only $\eta_{1}$ profiles for this complex.

The hardness and energy profiles along the linear transit path defined by the $\angle \mathrm{HOX}$ angle for the $\mathrm{HCCH} \cdots \mathrm{O}_{3}$ system do not follow the MHP. For all cases studied, the $\eta_{1}$ profiles have the same shape with a symmetric maximum connecting the two equivalent nonsymmetric minima. As before, the hardness profiles are more invariant with respect to the change of the basis set than the energy profiles. The lack of correspondence between the number of stationary points in the hardness and energy profiles calculated with the B3LYP/ 6-31G and $\mathrm{B} 3 \mathrm{LYP} / 6-311 \mathrm{G}(d, p)$ methods points out the shortcomings of the energy profiles at these levels of theory. The energy and $\eta_{1}$ profiles obtained at the B3LYP level with the STO-3G, 3-21G, 6-31+ G(d), 6-311++G, and $6-311 \mathrm{G}(d, p)$ basis sets are given as supporting information. While the $3-21 \mathrm{G}$ and $6-311 \mathrm{G}(d, p)$ basis sets yield wrong energy profiles showing only the spurious symmetric minimum, the $\eta_{1}$ profiles for all basis sets have always the correct number of stationary points.

\section{CONCLUSIONS}

In this work, we have studied the dependence of the energy and hardness profiles with respect to the level of calculation for a representative set of inter and intramolecular conformational changes. The chemical processes studied present energy profiles with spurious stationary points (i.e., the number and/or the type of the stationary points are 


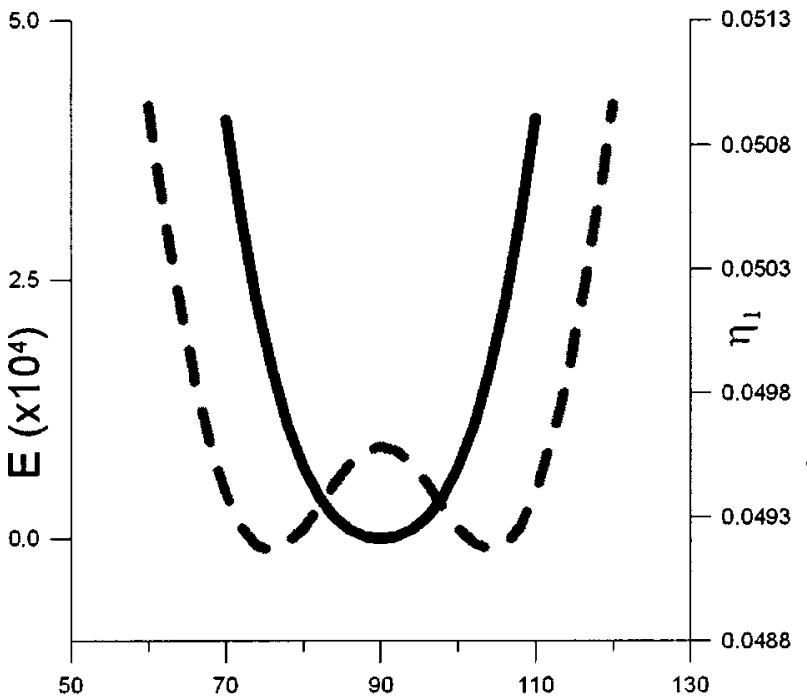

a) B3LYP/6-31G

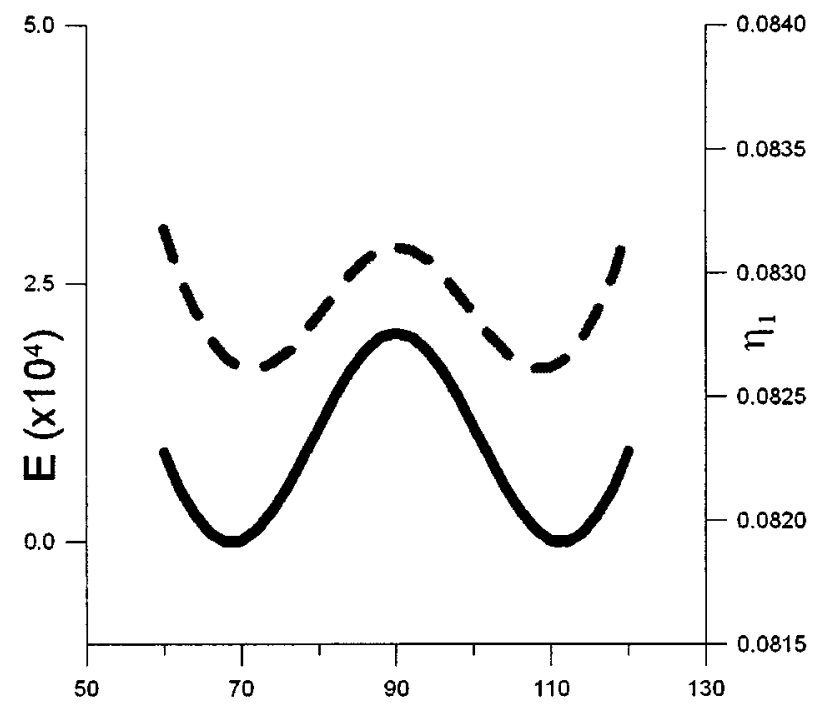

c) $B 3 L Y P / 6-311++G(d, p)$

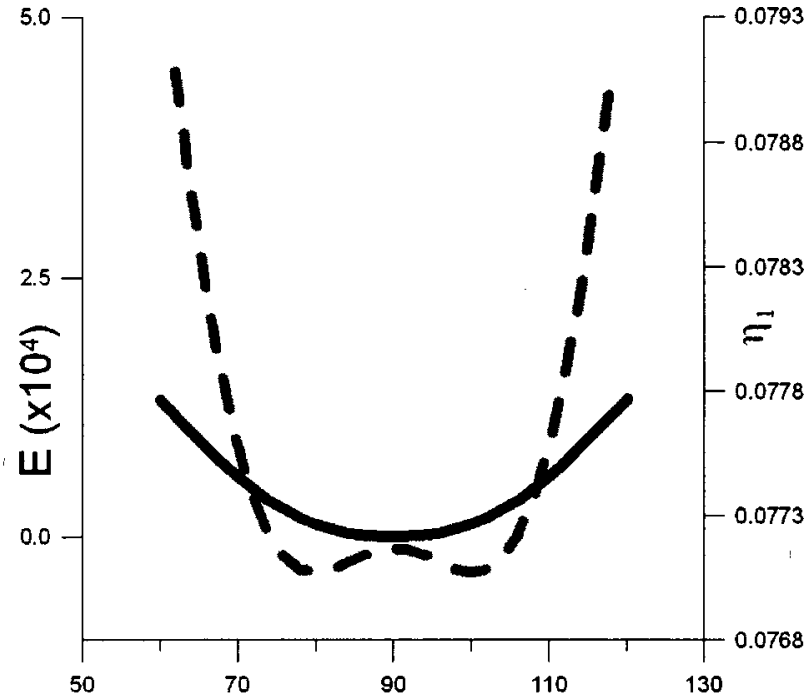

b) B3LYP/6-31G(d,p)

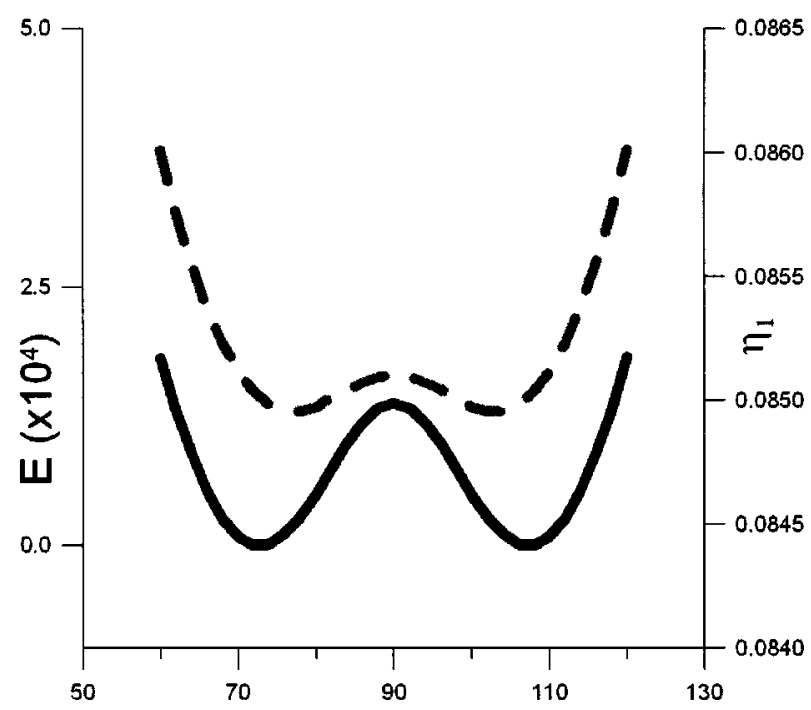

d) B3LYP/aug-cc-pVDZ

FIG. 7. Profiles of the relative energies (solid line) and hardness ( $\eta_{1}$ dashed line) calculated by changing the $\angle \mathrm{HOX}$ angle of the $\mathrm{HCCH} \cdots \mathrm{O}_{3}$ complex. The hardness values and the relative energies are given in a.u.

wrong) when the methodology used is not flexible enough to describe the subtle energetic changes involved in these processes. In all the examples investigated, the $\eta_{2}$ hardness profiles always show the correct number of stationary points. For $\eta_{1}$ hardness profile the only exception correspond to the linear transit path that connects the $C_{s}$ and $C_{2 v}$ stationary points in the $\mathrm{H}_{2} \mathrm{O} \cdots \mathrm{HCl}$ dimer. However, even in this case, the $\eta_{1}$ profiles lead also to the right number of stationary points when the linear transit path is replaced by the IRP. On the contrary, the energy profiles of the processes studied is found to be quite dependent on the method and basis set used. Although not discussed in the text, we have found that the polarizability profiles are even more sensitive to the change of the basis set than the energy profiles. The greater stability of the hardness profiles can be used to indicate the presence of spurious stationary points on the calculated po- tential energy surface in those chemical processes where the number of stationary points in the hardness and energy profiles does not coincide. In this sense, we think that the representation of the hardness profile could be a useful tool to check the validity of the energy profile for those chemical systems that because of their size can not be treated with high level ab initio methods (polymers, organic crystals, or biomolecules). Calculations on larger systems are necessary to further verify this new utility of the hardness profiles. Research in this direction is currently under way in our laboratory.

Supporting information available. The $E, \eta_{1}$, and $\eta_{2}$ profiles are provided for (a) the torsional rotation of $\mathrm{HOOH}$ computed at the B3LYP level with the STO-3G, 3-21G, 6-311G $(d, p), 6-311++\mathrm{G}(d, p)$, and aug-cc-pVTZ basis sets, and at the MP2 level with the 6-31G and 6-311+ 
$+\mathrm{G}(d, p)$ basis sets; (b) the rotation around the $\mathrm{B}-\mathrm{B}$ bond of $\mathrm{B}_{2} \mathrm{~F}_{4}$ calculated at the HF and MP2 levels with the 6-31G, 6-311G $(d)$, cc-pVDZ, and cc-pVTZ basis sets; (c) the $\angle \mathrm{SiCSi}$ angle variation computed with the $3-21 \mathrm{G}, 6-31 \mathrm{G}$, $6-31+\mathrm{G}(d), 6-311+\mathrm{G}$, and $6-311 \mathrm{G}(d)$ basis sets at the B3LYP level; (d) the $\angle \mathrm{HOH}_{1} \mathrm{X}$ angle variation of the $\mathrm{H}_{2} \mathrm{O} \cdots \mathrm{HCl}$ calculated at the B3LYP level with the STO-3G, $3-21 \mathrm{G}, 6-31+\mathrm{G}(d), 6-311 \mathrm{G}(d, p)$, and $6-311++\mathrm{G}(d, p)$ basis sets; (e) the $\angle \mathrm{HOX}$ angle variation of the $\mathrm{HCCH} \cdots \mathrm{O}_{3}$ computed with the STO-3G, 3-21G, 6-31+ G(d), 6-311+ $+\mathrm{G}$, and 6-311G $(d, p)$ basis sets at the B3LYP level, in this latter case only $E$ and $\eta_{1}$ profiles are depicted (see Ref. 67).

\section{ACKNOWLEDGMENTS}

Support for this work under Grants Nos. BQU200204112-C02-02 and BQU2002-03334 from the Dirección General de Enseñanza Superior e Investigación Científica y Técnica (MEC-Spain) is acknowledged. One of us (M.T.) thanks the Generalitat of Catalunya for financial help through CIRIT Project No. FI/01-00699. M.S. thanks the DURSI (Generalitat de Catalunya) for financial support through the Distinguished University Research Promotion, 2001. Excellent service and partial funding of computer time by the Center de Supercomputació de Catalunya (CESCA) is gratefully acknowledged. We are grateful to Dr. Pedro Salvador for helpful comments.

${ }^{1}$ H. B. Schlegel, Ab Initio Methods in Quantum Chemistry, edited by K. P. Lawley (Wiley, New York, 1987), p. 249.

${ }^{2}$ K. Fukui, Acc. Chem. Res. 14, 363 (1981).

${ }^{3}$ C. Gonzalez and H. B. Schlegel, J. Chem. Phys. 90, 2154 (1988).

${ }^{4}$ C. J. Cramer, Essentials of Computational Chemistry (Wiley, New York, 2002).

${ }^{5}$ H. B. Schlegel, J. Comput. Chem. 24, 1514 (2003).

${ }^{6}$ R. G. Parr and W. Yang, Density-Functional Theory of Atoms and Molecules (Oxford University Press, New York, 1989).

${ }^{7}$ R. G. Pearson, Chemical Hardness: Applications from Molecules to Solids (Wiley-VCH, Weinheim, 1997).

${ }^{8}$ R. G. Pearson, J. Chem. Educ. 64, 561 (1987); 76, 267 (1999).

${ }^{9}$ H. Chermette, J. Comput. Chem. 20, 129 (1999).

${ }^{10}$ P. Geerlings, F. De Proft, and W. Langenaeker, Chem. Rev. (Washington, D.C.) 103, 1793 (2003)

${ }^{11}$ T. K. Ghanty and S. K. Ghosh, J. Phys. Chem. 97, 4951 (1993).

${ }^{12}$ P. K. Chattaraj and S. Sengupta, J. Phys. Chem. 100, 16126 (1996).

${ }^{13}$ R. G. Parr and P. K. Chattaraj, J. Am. Chem. Soc. 113, 1854 (1991).

${ }^{14}$ D. Datta, J. Phys. Chem. 96, 2409 (1992).

${ }^{15}$ T. Kar and S. Scheiner, J. Phys. Chem. 99, 8121 (1995).

${ }^{16}$ P. K. Chattaraj, A. Cedillo, R. G. Parr, and E. M. Arnett, J. Org. Chem. 60, 4707 (1995).

${ }^{17}$ T. K. Ghanty and S. K. Ghosh, J. Phys. Chem. 100, 12295 (1996).

${ }^{18}$ T. Mineva, E. Sicilia, and N. Russo, J. Am. Chem. Soc. 120, 9053 (1998).

${ }^{19}$ A. Toro-Labbé, J. Phys. Chem. A 103, 4398 (1999).

${ }^{20} \mathrm{P}$. Jaque and A. Toro-Labbé, J. Phys. Chem. A 104, 995 (2000).

${ }^{21}$ P. Pérez and A. Toro-Labbé, J. Phys. Chem. A 104, 1557 (2000).

${ }^{22}$ P. K. Chattaraj, P. Fuentealba, B. Gómez, and R. Contreras, J. Am. Chem. Soc. 122, 348 (2000).

${ }^{23}$ U. Hohm, J. Phys. Chem. A 104, 8418 (2000).

${ }^{24}$ T. K. Ghanty and S. K. Ghosh, J. Phys. Chem. A 106, 4200 (2002).

${ }^{25}$ E. Sicilia, N. Russo, and T. Mineva, J. Phys. Chem. A 105, 442 (2001).

${ }^{26}$ T. Kar, S. Scheiner, and A. B. Sannigrahi, J. Phys. Chem. A 102, 5967 (1998).

${ }^{27}$ M. Solà and A. Toro-Labbé, J. Phys. Chem. A 103, 8847 (1999).

${ }^{28}$ L. T. Nguyen, T. N. Le, F. De Proft, A. K. Chandra, W. Langenaeker, M. T. Nguyen, and P. Geerlings, J. Am. Chem. Soc. 121, 5992 (1999).

${ }^{29}$ B. Gómez, P. K. Chattaraj, E. Chamorro, R. Contreras, and P. Fuentealba, J. Phys. Chem. A 106, 11227 (2002).
${ }^{30}$ A. K. Chandra and T. Uchimaru, J. Phys. Chem. A 105, 3578 (2001).

${ }^{31}$ B. Gómez, P. Fuentealba, and R. Contreras, Theor. Chem. Acc. 110, 421 (2003).

${ }^{32}$ M. Torrent-Sucarrat, J. M. Luis, M. Duran, and M. Solà, J. Chem. Phys. 117, 10561 (2002); J. Am. Chem. Soc. 123, 7951 (2001); Ll. Blancafort, M. Torrent-Sucarrat, J. M. Luis, M. Duran, and M. Solà, J. Phys. Chem. A 107, 7337 (2003).

${ }^{33}$ D. Cremer, J. Chem. Phys. 69, 4440 (1978); 69, 4456 (1978).

${ }^{34}$ Z.-H. Li and K.-N. Fan, J. Phys. Chem. A 106, 6659 (2002).

${ }^{35}$ R. S. Grev and H. F. Schaefer III, J. Chem. Phys. 82, 4126 (1984).

${ }^{36}$ M. M. Szczesniak, S. Scheiner, and Y. Bouteiller, J. Chem. Phys. 81, 5024 (1984); S. Re, Y. Osamura, Y. Suzuki, and H. F. Schaefer III, ibid. 109, 973 (1998).

${ }^{37}$ Z. Kisiel, B. A. Pietrewicz, P. W. Fowler, A. C. Legon, and E. Steiner, J. Phys. Chem. A 104, 6970 (2000).

${ }^{38}$ L. Turi and J. J. Dannenberg, J. Phys. Chem. 97, 7899 (1993).

${ }^{39}$ P. Salvador, S. Simon, M. Duran, and J. J. Dannenberg, J. Chem. Phys. 113, 5666 (2000).

${ }^{40}$ P. Salvador, X. Fradera, and M. Duran, J. Chem. Phys. 112, 10106 (2000).

${ }^{41}$ J.-M. Flaud, C. Camy-Peyret, J. W. C. Johns, and B. Carli, J. Chem. Phys. 91, 1504 (1989); S. Tommunphean, V. Parasuk, and A. Karpfen J. Phys. Chem. A 106, 438 (2002)

${ }^{42}$ I. M. B. Nielsen, W. D. Allen, A. G. Császár, and H. F. Schaefer III, J. Chem. Phys. 107, 1195 (1997).

${ }^{43}$ M. Shen, Y. Xie, H. F. Schaefer III, and C. A. Deakyne, J. Chem. Phys. 93, 3379 (1990); X.-B. Wang, X. Yang, L.-S. Wang, and J. B. Nicholas, ibid. 116, 561 (2002)

${ }^{44}$ N. L. Ma, S. S. Wong, M. N. Paddon-Row, and W.-K. Li, Chem. Phys. Lett. 213, 189 (1993).

${ }^{45}$ S. Tsuzuki, T. Uchimaru, M. Mikami, and K. Tanabe, J. Chem. Phys. 109, 2169 (1998)

${ }^{46}$ E. F. Valeev and H. F. Schaefer III, J. Chem. Phys. 108, 7197 (1998).

${ }^{47}$ D. J. Giesen and J. A. Phillips, J. Phys. Chem. A 107, 4009 (2003); D. L. Fiacco and K. R. Leopold, ibid. 107, 2808 (2003).

${ }^{48}$ S. Simon, M. Duran, and J. J. Dannenberg, J. Chem. Phys. 105, 11024 (1996).

${ }^{49}$ F. De Proft and P. Geerlings, J. Chem. Phys. 106, 3270 (1997).

${ }^{50}$ J. P. Perdew and R. G. Parr, Phys. Rev. Lett. 49, 1691 (1982).

${ }^{51}$ L. Komorowski, Chem. Phys. Lett. 103, 201 (1983); 134, 536 (1987).

${ }^{52}$ S. Liu, F. De Proft, and R. G. Parr, J. Phys. Chem. A 101, 6991 (1997); M. Torrent-Sucarrat, M. Duran, and M. Solà, ibid. 106, 4632 (2002).

${ }^{53}$ R. Balawender and L. Komorowski, J. Chem. Phys. 109, 5203 (1998).

${ }^{54}$ R. F. Nalewajski, Chemical Hardness, edited K. D. Sen (Springer-Verlag, Berlin, 1993), p. 194.

${ }^{55}$ T. Koopmans, Physica (Utrecht) 1, 104 (1934).

${ }^{56}$ C. C. J. Roothann, Rev. Mod. Phys. 35, 65 (1951)

${ }^{57}$ A. D. Becke, J. Chem. Phys. 98, 5648 (1993); C. Lee, W. Yang, and R. G. Parr, Phys. Rev. B 37, 785 (1988); P. J. Stevens, F. J. Devlin, C. F. Chablowski, and M. J. Frisch, J. Phys. Chem. 98, 11623 (1994).

${ }^{58}$ C. Møller and M. S. Plesset, Phys. Rev. 46, 618 (1934).

${ }^{59}$ W. J. Hehre, L. Radom, P. v. R. Schleyer, and J. A. Pople, Ab Initio Molecular Orbital Theory (Wiley, New York, 1986).

${ }^{60}$ T. H. Dunning Jr., J. Chem. Phys. 90, 1007 (1989); R. A. Kendall, T. H. Dunning Jr., and R. J. Harrison, ibid. 96, 6796 (1992); D. E. Woon and T. H. Dunning Jr., ibid. 98, 1358 (1993).

${ }^{61}$ M. J. Frisch, G. W. Trucks, H. B. Schlegel et al., Gaussian 98, Revision A.11, Gaussian, Inc., Pittsburgh, Pennsylvania, 2001.

${ }^{62}$ P. A. Giguère and T. K. K. Srinivasan, J. Mol. Spectrosc. 66, 168 (1977).

${ }^{63}$ P. K. Chattaraj, P. Fuentealba, P. Jaque, and A. Toro-Labbé, J. Phys. Chem. A 103, 9307 (1999).

${ }^{64}$ G. Makov, J. Phys. Chem. 99, 9337 (1995)

${ }^{65}$ At the MP2 level, $\eta_{1}$ is calculated using the HOMO and LUMO energies of the Hartree-Fock molecular orbitals.

${ }^{66}$ Z. H. Kafafi, R. H. Hauge, L. Fredin, and J. L. Margrave, J. Phys. Chem. 87, 797 (1983).

${ }^{67}$ See EPAPS Document No. E-JCPSA6-120-303423 for the $E$, $\eta_{1}$, and $\eta_{2}$ profiles studied but not included in this paper. A direct link to this document may be found in the online article's HTML reference section. The document may also be reached via the EPAPS homepage (http:// www.aip.org/pubservs/epaps.html) or from ftp.aip.org in the directory lepaps/. See the EPAPS homepage for more information. 